08

\title{
Диэлектрическая спектроскопия сильно коррелированных электронных состояний диоксида ванадия
}

\author{
(C) А.В. Ильинский, ${ }^{1}$ Р.А. Кастро, ${ }^{2}$ Е.И. Никулин, ${ }^{1}$ Е.Б. Шадрин ${ }^{1}$ \\ ${ }^{1}$ Физико-технический институт им. А.Ф. Иоффре РАН, \\ 194021 Санкт-Петербург, Россия \\ ${ }^{2}$ Российский государственный педагогический университет им. А.И. Герцена, \\ 191186 Санкт-Петербург, Россия \\ e-mail: ilinskiy@mail.ioffe.ru
}

(Поступило в Редакцию 18 июля 2017 г.)

В интервале температур $-196<T<100^{\circ} \mathrm{C}(77<T<273 \mathrm{~K})$ прослежена термическая эволюция проводимости пленки $\mathrm{VO}_{2}$ и полученной на базе данных ширины $E_{g}$ запрещенной зоны нанокристаллитов пленки; определена глубина залегания примесных центров донорного типа $\left(E_{d}=0.04 \mathrm{eV}\right)$. Показано, что с ростом температуры в интервале $273<T<300 \mathrm{~K}$ энергия $E_{g}$ уменьшается от 0.8 до $\sim 0 \mathrm{eV}$, что обусловлено сужением энергетической щели благодаря корреляционным эффектам и позиционируется как протяженный по температуре моттовский электронный фазовый переход „диэлектрик-металл“ с сохранением моноклинной симметрии решетки. Последующий скачок симметрии от моноклинной до тетрагональной при дальнейшем росте температуры позиционируется как структурный фазовый переход Пайерлса, температура которого лежит в области $340 \mathrm{~K}$ и определяется размерными эффектами, нестехиометрией нанокристаллитов пленки $\mathrm{VO}_{2}$ и степенью их адгезии к подложке.

DOI: 10.21883/JTF.2018.06.46019.2437

\section{Введение}

Настоящая работа посвящена изучению электронных состояний кристаллических пленок диоксида ванадия методами диэлектрической спектроскопии. Данные методы широко применяются при исследовании частотных и температурных изменений параметров различных материалов [1-3], в связи с чем представляется разумным использовать методы диэлектрической спектроскопии для экспресс-анализа свойств сильно коррелированных материалов, в частности, для изучения характеристик моттовской части комплексного фазового перехода (ФП) диэлектрик-металл в таком сильно коррелированном материале, как диоксид ванадия. Достоинством методики диэлектрической спектроскопии является ее высокая чувствительность к изменению состояния нанокристаллических материалов, что открывает возможность высокоточного экспресс-измерения электрофизических параметров предельно тонких окиснованадиевых пленок (толщиной менее $100 \mathrm{~nm}$ ).

Следует отметить, что исследование пленок диоксида ванадия оправдано как с прикладной, так и с фундаментальной точек зрения [4-6]. Последнее обусловлено совершением вблизи $T_{c}$ двух типов ФП: электронного перехода Мотта и структурного перехода Пайерлса, которые происходят в различных нанокристаллитах пленки $\mathrm{VO}_{2}$ при несколько различных температурах, определяемых вариацией строения кристаллитов. По этой причине модель механизма ФП достаточно сложна и включает в себя как учет многочастичного взаимодействия электронов (корреляционных эффектов), так и образований типа димеров из атомов ванадия, принадлежащих соседним элементарным кристаллическим ячейкам.

Сказанное определяет необходимость детального изучения всей совокупности перечисленных явлений, что в настоящей работе выполнено путем исследования термической зависимости электропроводности пленок $\mathrm{VO}_{2}$ в широком интервале температур $\left(-196<T<100^{\circ} \mathrm{C}\right.$, $77<T<373 \mathrm{~K})$. Это позволяет проследить процесс термического схлопывания запрещенной зоны материала вследствие корреляционных эффектов.

\section{Образцы и методика эксперимента}

Тонкопленочные структуры на основе нанокристаллов диоксида ванадия были получены методом лазерной абляции при бомбардировке $V$-мишени излучением импульсного ИК-лазера на алюмоиттриевом гранате с неодимом. Плотность мощности излучения составляла $10^{7}-10^{9} \mathrm{~W} / \mathrm{cm}^{2}$, длительность импульса излучения была равна $20 \mathrm{~ns}$. Температура подложки в процессе синтеза поддерживалась в интервале $400-650^{\circ} \mathrm{C}$. Давление кислорода в камере варьировало в пределах от $10^{-2}$ до $2 \cdot 10^{-1}$ Torr. Это давало возможность синтезировать нанокристаллические слои диоксида ванадия с зернами разного размера. Морфология пленки $\mathrm{VO}_{2}$ контролировалась путем анализа атомно-силовых изображений пленки (рис. 1).

Площадь поверхности пленочных образцов составляла $10 \times 10 \mathrm{~mm}^{2}$, толщина пленок $1100 \AA$, удельное сопротивление $7 \cdot 10^{6} \Omega \cdot \mathrm{m}$ при комнатной температуре.

Обращает на себя внимание тот факт, что пленка $\mathrm{VO}_{2}$ состоит из одного слоя примыкающих друг к 


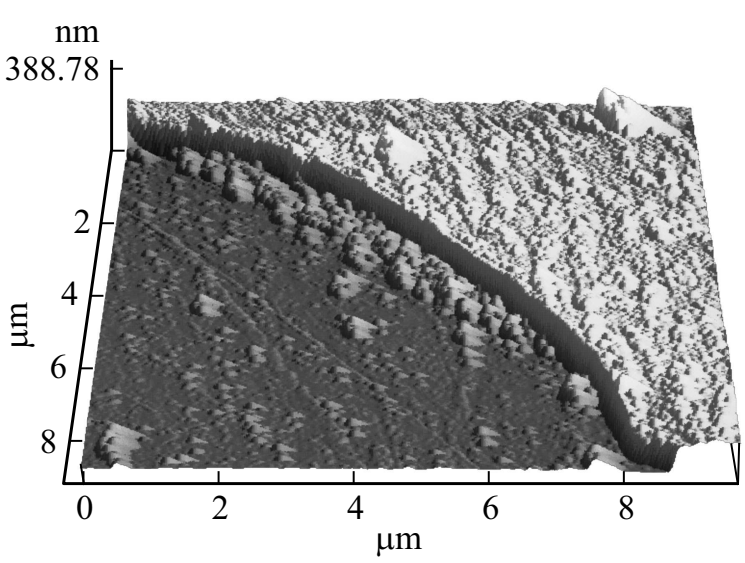

Рис. 1. Атомно-силовое изображение поверхности пленки и ее торца, полученного методом локального травления пленки.

другу столбчатых нанокристаллитов, которые контактируют между собой боковыми гранями (рис. 1). Данный рисунок демонстрирует боковой срез монослоя $\mathrm{VO}_{2}$ кристаллитов в виде ступеньки, полученной методом травления.

Исследования диэлектрических спектров и их температурная зависимость выполнялись на диэлектрическом спектрометре марки „Alpha-Beta Impedance Analyzer“ фирмы Novocontrol Technologies. Образец пленки диоксида ванадия, синтезированной на ситалловой подложке, помещался между плоскими электродами диаметром $20 \mathrm{~mm}$. Измерялась амплитуда перпендикулярно поверхности пленки тока через образец $I_{0}$ при подаче на него эталонного синусоидального напряжения $U(t)$. Измерялась также разность фаз $\varphi$ колебаний $U(t)$ и $I(t)$. С помощью конвертера эти данные с применением разработанных фирмой Novocontrol Technologies компьютерных программ преобразовывались в данные о физических характеристиках образца $\left(\operatorname{tg} D, \varepsilon^{\prime}, \varepsilon^{\prime \prime}\right)$.

Измерения были выполнены на 10 дискретных частотах $f$ в интервале от $10 \mathrm{kHz}$ до $1 \mathrm{MHz}$ с постоянным логарифмическим шагом, температура образца $T$ непрерывно изменялась в процессе измерений от -100 до $+100^{\circ} \mathrm{C}$ (от 173 до $373 \mathrm{~K}$ ) с постоянной скоростью $5^{\circ} \mathrm{C} / \mathrm{min}$. Алгоритм температурных измерений состоял в следующем: нанокристаллическая пленка $\mathrm{VO}_{2}$ изначально нагревалась от комнатной температуры до температуры $+100^{\circ} \mathrm{C}(373 \mathrm{~K})$. После этого производилось охлаждение пленки до температуры $-100^{\circ} \mathrm{C}$ $(173 \mathrm{~K})$ и после этого вновь производился нагрев до $+100^{\circ} \mathrm{C}(373 \mathrm{~K})$.

Были также выполнены контрольные измерения проводимости пленок в планарной геометрии стандартным четырехзондовым методом, в котором к пленке с помощью нанесенных на нее электродов прикладывалось постоянное напряжение, и измерялся ток, протекающий вдоль поверхности пленки.

\section{Результаты эксперимента и их обработка}

На рис. 2 представлены частотные зависимости тангенса угла диэлектрических потерь нанокристаллической пленки $\mathrm{VO}_{2}$ при различных температурах в диапазоне от -100 до $100^{\circ} \mathrm{C}$ (от 173 до $373 \mathrm{~K}$ ).

На рис. 3 представлена простейшая эквивалентная электрическая схема образца и расчетная кривая частотной зависимости $\operatorname{tg} D$ для такой схемы.

При анализе экспериментальных данных предполагалось, что эквивалентной схемой является параллельное соединение сопротивления $R$ и электроемкости $C_{a} \mathrm{c}$ включением последовательно с этой парой элементов дополнительной емкости $C_{b}$. Такая наиболее простая схема с необходимостью включает в себя наличие электрических емкостей, одна из которых $\left(C_{a}\right)$ является электроемкостью пленки $\mathrm{VO}_{2}$, запараллеленной сопротивлением $(R)$ пленки, а другая $\left(C_{b}\right)$ представляет собой электрическую емкость ситалловой подложки. Расчеты, выполненные на основе данной электрической эквивалентной схемы, позволяют получить в отличие от
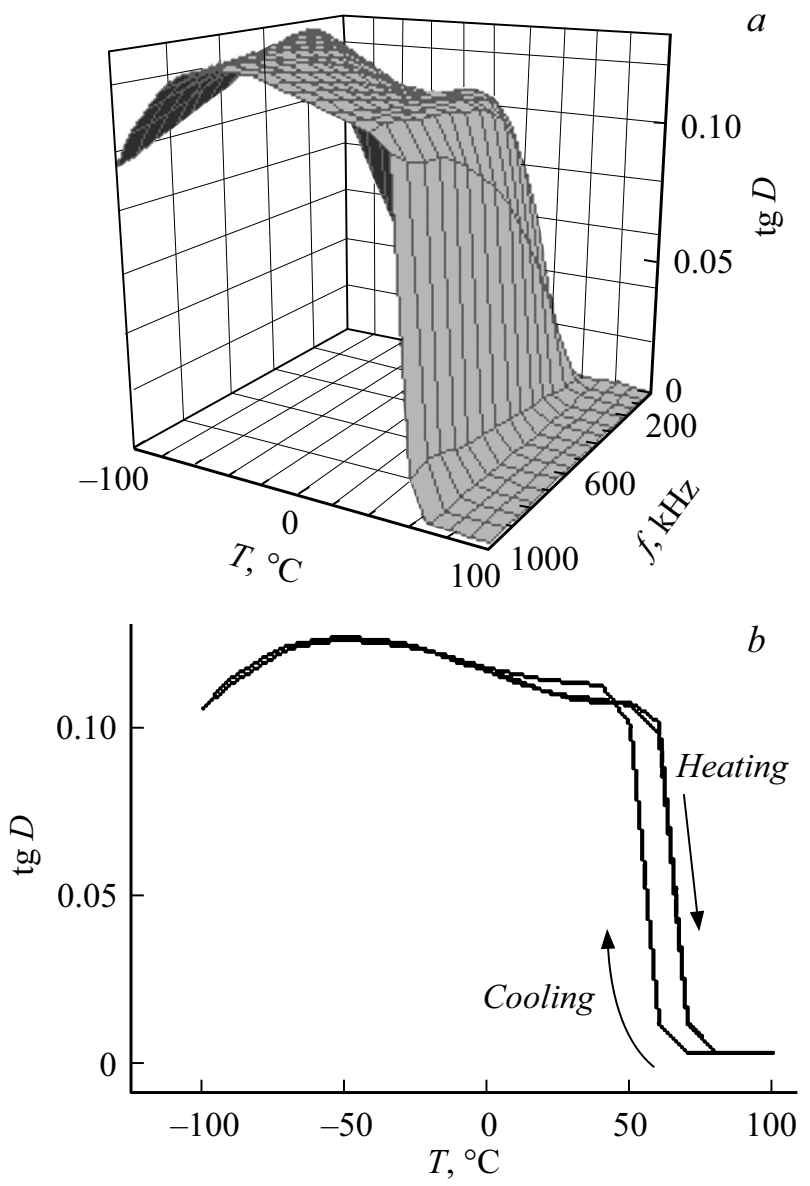

Pис. 2. Диэлектрические спектры $\operatorname{tg} D$ (частотные зависимости тангенса угла диэлектрических потерь) при различных температурах в диапазоне от -100 до $100^{\circ} \mathrm{C}$ нанокристаллической пленки $\mathrm{VO}_{2}(a)$; две ветви (нагревная и охладительная) температурной зависимости $\operatorname{tg} D$ для частоты $714286 \mathrm{~Hz}(b)$. 


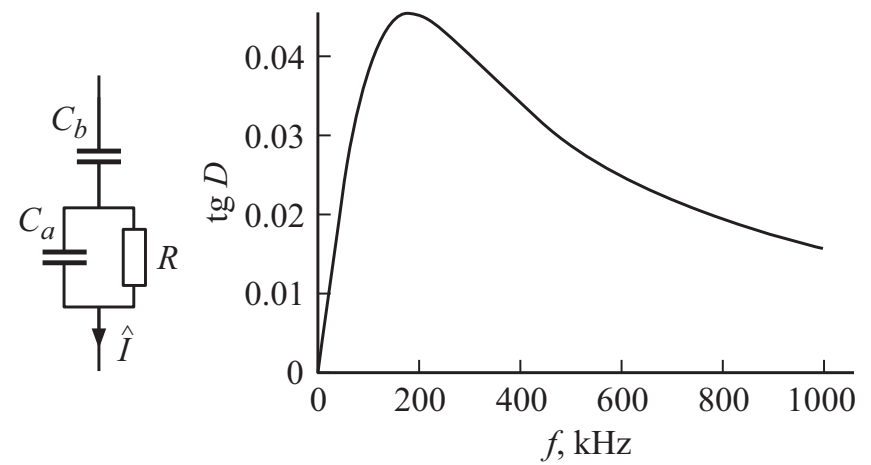

Рис. 3. Эквивалентная схема образца и расчетная частотная зависимость $\operatorname{tg} D$, полученная для значений $C_{a}=170 \mathrm{pF}$, $C_{b}=16 \mathrm{pF}, R=5 \mathrm{k} \Omega$.

более простых схем, содержащих лишь одну электроемкость вместо двух, максимум на частотной зависимости тангенса диэлектрических потерь, что соответствует эксперименту (рис. 2, $a$ ).

Аналитическое выражение тангенса угла диэлектрических потерь для эквивалентной схемы рис. 3 имеет вид

$$
\operatorname{tg} D=\left[(2 \pi f) R C_{b}\right] /\left[(2 \pi f)^{2}\left(C_{a}\right)^{2} R^{2}\left(C_{b} / C_{a}+1\right)+1\right] .
$$

На рис. 3 представлена частотная зависимость $\operatorname{tg} D$, полученная по этой формуле.

Фитинг функциональной зависимости тангенса угла диэлектрических потерь от величин $C_{a}, C_{b}$ и $R$, даваемой формулой (1), на экспериментальные данные дает хорошее согласие с результатами, представленными на рис. 2, при постоянных, не зависящих от частоты и температуры, значениях емкостей $C_{b}=16 \mathrm{pF}, C_{a}=170 \mathrm{pF}$. Варьируемым при фитинге параметром является сопротивление образца $R$ при разных температурах. Полученные данные позволяют построить температурную зависимость сопротивления $R$ пленочного образца $\mathrm{VO}_{2}$ в предположении, что численные значения $C_{a}, C_{b}$ и $R$ не являются функциями частоты.

На рис. 4, $a$ представлены полученные методом фитинга температурные зависимости сопротивления, вычисленные на основе данных рис. 2, при фиксированных значениях емкости подложки $\left(C_{b}=16 \mathrm{pF}\right)$, на которую нанесена пленка, и самой пленки $\left(C_{a}=170 \mathrm{pF}\right)$. На pис. 4, $b$ представлена полученная на основе данных pис. 4, $a$ зависимость логарифма проводимости $\sigma=1 / R$ пленки диоксида ванадия от обратной температуры $1 / T$ (координаты Аррениуса).

Анализ низкотемпературного участка 1 (рис. 4,b) дает $\ln [n(T)]=E_{g} / k T+$ const. В координатах Аррениуса это позволяет определить энергию активации, оказавшуюся равной $E_{g}=0.8 \mathrm{eV}$. Обращает на себя особое внимание тот факт, что с повышением температуры (рис. $4, b)$ наклон кривой $\ln \sigma(1 / T)$ уменьшается от значения $0.8 \mathrm{eV}$ практически до нуля, причем последнее значение достигается при температуре примерно $0^{\circ} \mathrm{C}$ (в области $273 \mathrm{~K})$, т. е. до совершения структурного ФП Пайерлса при различных температурах $T \sim 50-90^{\circ} \mathrm{C}$ $(\sim 320-360 \mathrm{~K})$ в различных зернах пленки $\mathrm{VO}_{2}$.

Результаты непосредственного измерения при различных температурах сопротивления пленки на постоянном токе, текущем вдоль поверхности пленки (контрольных измерений), приведены на рис. 5.

Низкотемпературный участок 2 кривой $\ln \sigma(T)$ рис. $5, b$ отвечает случаю термической ионизации мелких доноров электронов [7]. Для данного низкотемпературного участка из выражения

$$
n_{1}(T)=n_{1}(0) \exp \left(-E_{d} / k T\right)
$$

следует $\ln \left[n_{1}(T)\right]=-E_{d} / k T+$ const. Использование координат Аррениуса приводит к линеаризации указанного низкотемпературного участка, что позволяет по тангенсу угла его наклона к оси абсцисс определить энергию ионизации доноров $E_{d}=0.04 \mathrm{eV}$.

Данным стандартным методом получены аналогичные результаты и для ширины запрещенной зоны $E_{g}$. Здесь хорошо видно, что с повышением температуры (рис. $5, b)$ наклон кривой $\ln \sigma(1 / T)$ уменьшается от значения $E_{g}=0.8 \mathrm{eV}$ практически до нуля, причем вновь
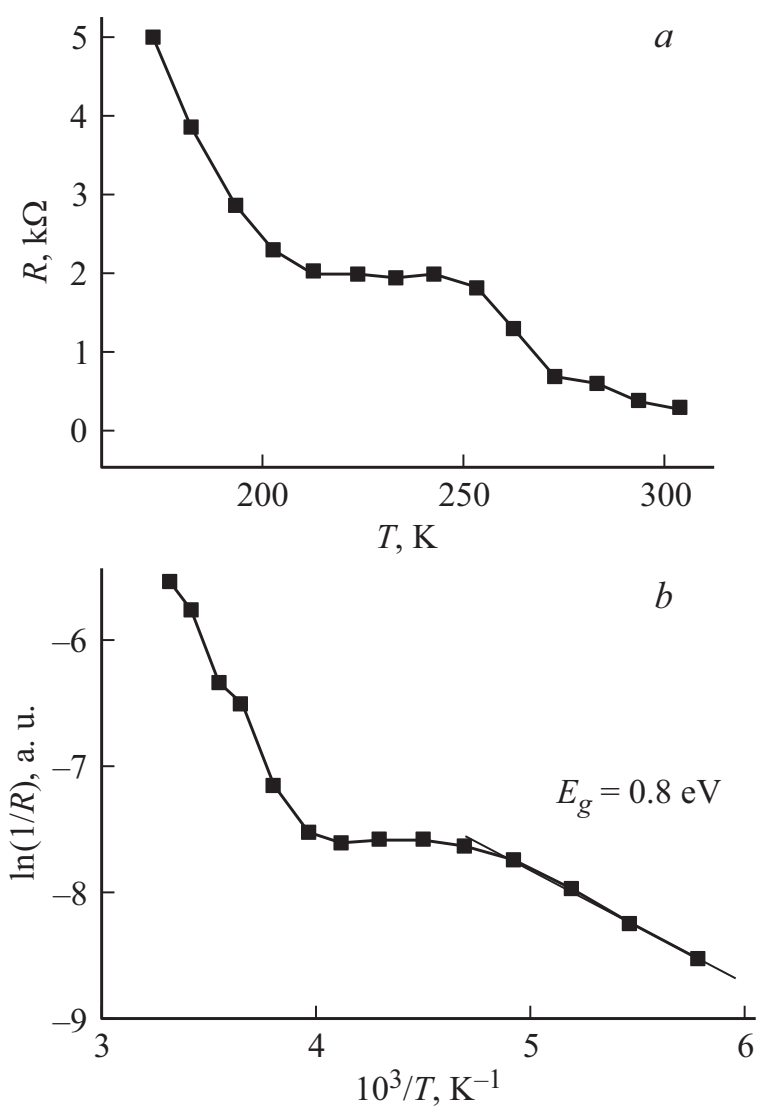

Рис. 4. Температурная зависимость сопротивления $R(T)$ пленки $\mathrm{VO}_{2}$, полученная методами диэлектрической спектроскопии $(a)$, и построенная из этих данных зависимость логарифма проводимости $\ln (1 / R)$ от обратной температуры (координаты Аррениуса) (b). 

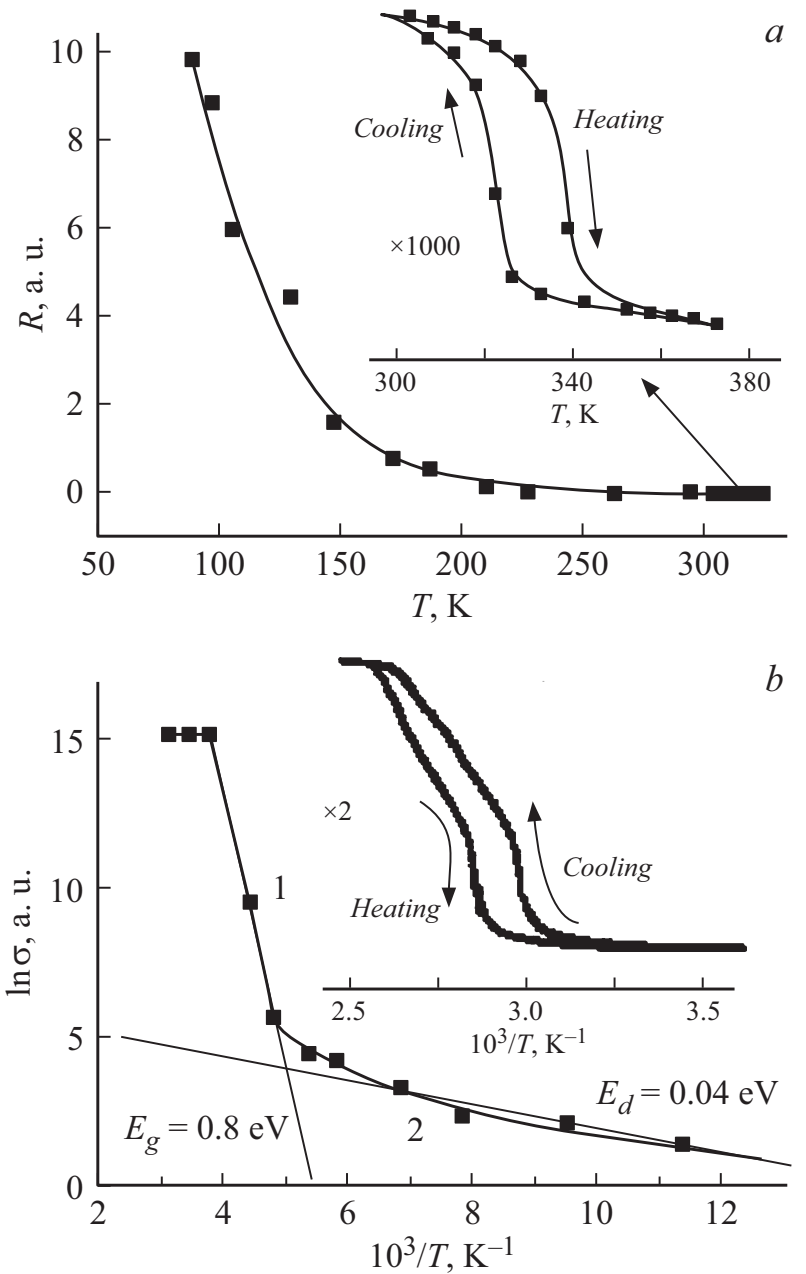

Рис. 5. Температурная зависимость сопротивления $R(T)$ пленки $\mathrm{VO}_{2}$, полученная при протекании постоянного тока вдоль поверхности пленки между нанесенными на ее поверхность электродами $(a)$, и построенная на базе этих данных зависимость логарифма проводимости $\ln (1 / R)$ от обратной температуры (координаты Аррениуса) (b). На вставках приведены петли температурного гистерезиса $R$ и $\ln (1 / R)$ в области структурного ФП.

при температуре приблизительно $0^{\circ} \mathrm{C}(273 \mathrm{~K})$. Такое уменьшение, как будет показано ниже, обусловлено корреляционными эффектами в диоксиде ванадия.

Заметим, что описанный эксперимент дает значение $\sigma$, зависящее от температуры и изменяющееся в пределах от $\sigma=10^{-6}$ до $10^{-7} \Omega^{-1} \cdot \mathrm{m}^{-1}$. Это соответствует изменению максвелловского времени релаксации в пределах $\tau_{\mathrm{M}}=3.56 \cdot 10^{-3} \mathrm{~s}$ до величины $\tau_{\mathrm{M}}=3.56 \cdot 10^{-4} \mathrm{~s}$. Отсюда следует, что для зондирующих частот, больших $1 / \tau_{\mathrm{M}}$, можно использовать формулу (1). А для частот, равных и меньших $1 / \tau_{\mathrm{M}}$, эквивалентная схема рис. 3 должна быть существенно усложнена, так как в этом случае необходимо принимать во внимание изменение емкости области пространственного заряда [8] в течение периода колебаний прикладываемого напряжения. По указанной причине фитинг экспериментальной кривой рис. 4 осуществлялся в настоящей работе только для области высоких частот от значения $f=\left(1 / \tau_{\mathrm{M}}\right)=30 \mathrm{kHz}$.

Обратим внимание также на то, что фитинг частотных зависимостей $\operatorname{tg} D$ осуществлялся лишь в области температур от -100 до $+40^{\circ} \mathrm{C}$ (от 173 до $313 \mathrm{~K}$ ), т.е. в области, соответствующей протяженному по температуре корреляционному переходу Мотта [9]. А фитинг при температурах $40-100^{\circ} \mathrm{C}(313-373 \mathrm{~K})$ не производился. Это связано с тем, что при пайерлсовском структурном ФП, совершающемся в области температур $40-100^{\circ} \mathrm{C}$ $(313-373 \mathrm{~K})$, проводимость пленочного образца скачкообразно возрастает на 3-4 порядка. Таким же образом возрастает частота, соответствующая $1 / \tau_{\mathrm{M}}$, значение которой оказывается гораздо большим $1 \mathrm{MHz}$ - предельной рабочей частоты экспериментальной установки. Таким образом, указанная область фитинга оказывается недоступной.

В заключение укажем, что выполненные в настоящей работе эксперименты показывают, что сильные электрон-электронные взаимодействия обнаруживают себя также и в спектрах комбинационного рассеяния света (КРС) нанокристаллитами пленки $\mathrm{VO}_{2}$ в виде появления широкого корреляционного максимума. Развитие этого максимума проявляется с ростом температуры

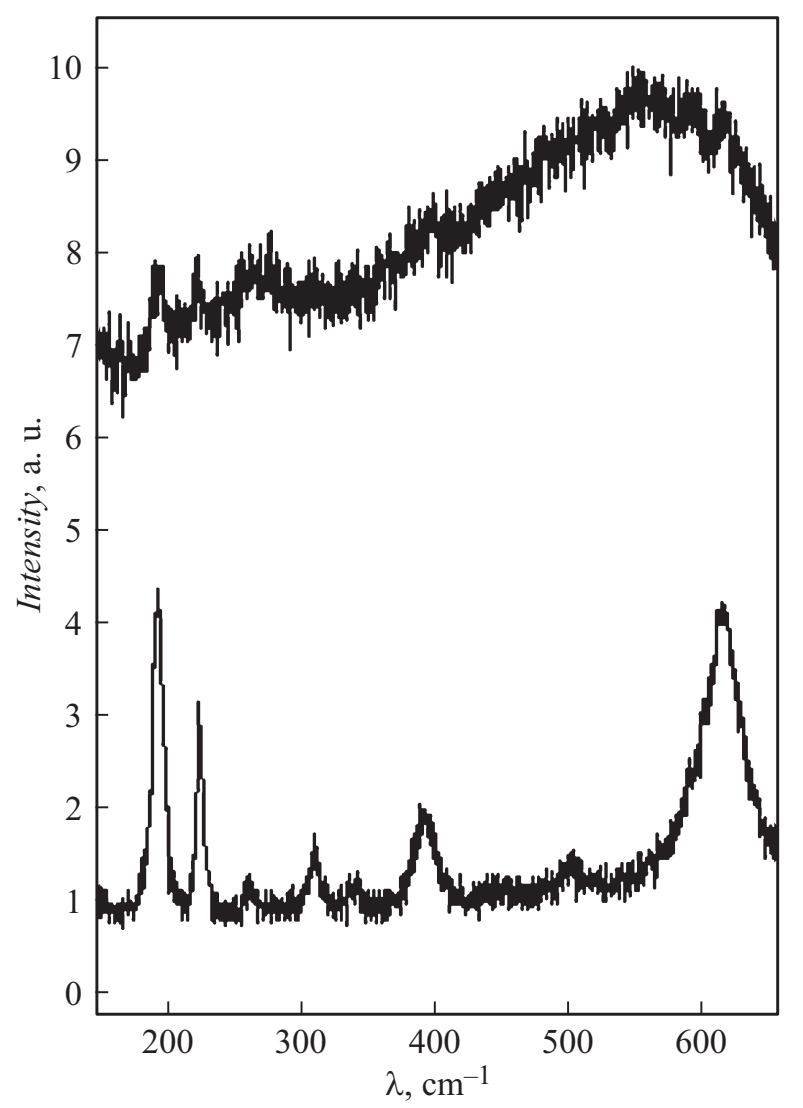

Рис. 6. Спектры КРС пленки $\mathrm{VO}_{2}$, полученные при $T=293 \mathrm{~K}$ (нижняя кривая) и при $T=336 \mathrm{~K}$ (верхняя кривая). 
в перекачке в него силы осциллятора узких фононных пиков диэлектрической моноклинной фазы $\mathrm{VO}_{2}$.

На рис. 6 представлен спектры КРС $\mathrm{He}-\mathrm{Ne}$-лазера пленкой $\mathrm{VO}_{2}$ при $T=20$ и $63^{\circ} \mathrm{C}(293$ и $336 \mathrm{~K})$.

Действительно, как показано на рис. 6, спектр КРС в стоксовой области при комнатной температуре $T=20^{\circ} \mathrm{C}(293 \mathrm{~K})$ представляет собой систему узких фононных линий, характеристики которых соответствуют литературным данным для моноклинной диэлектрической фазы [10]. В то же время при повышении температуры в спектре КРС диэлектрической фазы $\mathrm{VO}_{2}$ ясно прослеживается постепенное возгорание широкого максимума, появление первых признаков которого наблюдается уже при температуре более чем на $20^{\circ} \mathrm{C}$ ниже точки $T_{c}=67^{\circ} \mathrm{C}(340 \mathrm{~K})$ структурного пайерлсовского перехода, т.е. при температуре $T=47^{\circ} \mathrm{C}(320 \mathrm{~K})$. На рис. 6 представлен спектр КРС изолирующей фазы $\mathrm{VO}_{2}$ при $T=63^{\circ} \mathrm{C}(336 \mathrm{~K})$, т. е. в непосредственной близости от $T_{c}=67^{\circ} \mathrm{C}(340 \mathrm{~K})$. Температура $T=63^{\circ} \mathrm{C}(336 \mathrm{~K})$ соответствует состоянию, при котором уже наблюдается развитый широкий максимум около $320 \mathrm{~cm}^{-1}$, на фоне которого располагаются хорошо регистрируемые узкие линии спектра КРС диэлектрической фазы. При дальнейшем повышении температуры, т.е. за точкой структурного ФП, этот максимум продолжает возрастать и, кроме того, изменяет свою форму, расщепляясь на две слабо разрешенные полосы с центрами при 230 и $450 \mathrm{~cm}^{-1}$. Усредненное положение данного максимума соответствует частоте $320 \mathrm{~cm}^{-1}$.

\section{Обсуждение результатов}

Вся совокупность наблюдаемых явлений может быть объяснена на основе следующих физических соображений.

Для диоксида ванадия корреляционные эффекты, согласно многостадийной схеме ФП в $\mathrm{VO}_{2}$ [9], приводят при нагреве образца к уменьшению энергетической щели между нижней $3 d \|$-подзоной и $\pi^{*}$-зоной (рис. 7).

В каждом нанокристаллите пленки в интервале температур от -50 до $+20^{\circ} \mathrm{C}$ (от 223 до $293 \mathrm{~K}$ ) ширина запрещенной зоны изменяется от стартовой величины $0.8 \mathrm{eV}$ практически до $0 \mathrm{eV}$ за счет корреляционного движения зон, что представляет собой протяженный по температуре электронный переход Мотта. Движение зон вызвано чувствительностью энергетического положения зон к термическому забросу электронов из нижней $3 d \|$ подзоны в $\pi^{*}$-зону, поскольку в сильно коррелированных материалах энергетическое положение зон обратно пропорционально их заселенности [11]. Симметрия решетки при электронном ФП Мотта не изменяется, оставаясь моноклинной, характерной для диэлектрической фазы $\mathrm{VO}_{2}$. Скачок симметрии до тетрагональной в каждом отдельном зерне пленки происходит лишь при дальнейшем ее нагреве в интервале $50-80^{\circ} \mathrm{C}(323-353 \mathrm{~K})$ за счет совершения ФП Пайерлса. Скачок в разных

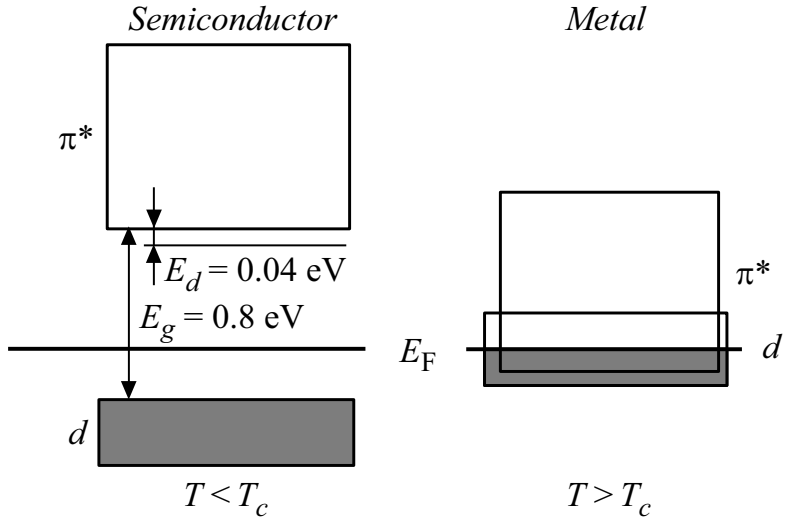

Рис. 7. Эволюция энергетической схемы диоксида ванадия при ФП диэлектрик-металл.

зернах происходит при разных температурах, зависящих от размера зерна [9]. Согласно многостадийной схеме, механизм ФП диэлектрик-металл в $\mathrm{VO}_{2}$ состоит в следующем.

Корреляционное взаимодействие между электронами, следуя за термическим ростом заселенности зоны проводимости и соответственно ростом степени опустошения валентной зоны, сужает энергетическую щель $0.8 \mathrm{eV}$, т. е. запрещенную зону, вплоть до соприкосновения дна $\pi^{*}$ зоны (играющей роль зоны проводимости) с вершиной нижней $3 d \|$-подзоны (играющей роль валентной зоны) на уровне Ферми (рис. 7). Этот процесс является электронным переходом Мотта, протяженным по температуре в широком интервале. При дальнейшем нагреве все ниже опускающаяся $\pi^{*}$-зона проходит уровень Ферми своими различными энергетическими слоями, что приводит к интенсификации переходов электронов из нижней $3 d \|$-подзоны в $\pi^{*}$-зону в соответствии с формой функции распределения электронных состояний [11] и постепенному разрушению $\sigma$-связей димеров [12], электронные состояния которых формируют нижнюю $3 d \|-$ подзону. Продолжающийся нагрев сопровождается продолжающимся ростом относительной доли разрушенных $\sigma$-связей $V V$-димеров, которая в свою очередь определяется приближением при опускании максимума функции плотности электронных состояний $\pi$-зоны к уровню Ферми [9]. Полное скачкообразное разрушение всех димеров решетки, требуемое для совершения структурного перехода Пайерлса, происходит при перетекании критической части электронов $3 d \|$-подзоны в $\pi^{*}$-зону. Это принципиально возможно лишь в том случае, если число состояний в $\pi^{*}$-зоне существенно больше, чем в нижней $3 d \|$-подзоне. Данное условие для $\mathrm{VO}_{2}$ жестко выполняется, так как число уровней в нижней $3 d \|$ подзоне равно $N / 2$, а в $\pi^{*}$-зоне оно равно $2 N$, где $N-$ число атомов ванадия. Одновременно с концентрационным движением зон корреляционное взаимодействие многократно расширяет „хвосты“ распределения электронов по уровням энергии, уменьшая соответственно 
величину на уровне Ферми скачка функции распределения электронов по энергиям и превращая его в скачок Мигдала [13]. При достижении (при $T=T_{c}$ ) критической концентрации разрушенных $\sigma$-связей димеров в каждом нанокристаллите пленки $\mathrm{VO}_{2}$ происходит структурный ФП в тетрагональную фазу, причем происходит скачкообразно при $T=T_{c}+\Delta T$, где $T_{c}-$ температура равновесия контактирующих фаз, а $\Delta T$ - полуширина элементарной петли гистерезиса нанокристаллита [14].

О том, что корреляционные эффекты, связанные с ФП Мотта, дают о себе знать „задолго“ до совершения структурного ФП Пайерлса, происходящего в каждом отдельном зерне пленки при своей температуре (варьирующей в интервале от 55 до $68^{\circ} \mathrm{C}$ ), свидетельствует, как указывалось, наряду с данными диэлектрической спектроскопии, термическая трансформация спектров КРС (рис. 6). В этих спектрах, подчеркнем еще раз, при $0^{\circ} \mathrm{C}(273 \mathrm{~K})$ наблюдаются только узкие линии КРС диэлектрической фазы, которые обусловлены взаимодействием рассеянного света с фононной подсистемой моноклинной фазы. Но уже при $0^{\circ} \mathrm{C}(273 \mathrm{~K})$, когда начинается корреляционное сужение запрещенной зоны и появляются свободные коррелированные электроны, в спектре КРС появляется, а при более высоких температурах и интенсивно возгорается новый широкий максимум $\left(\sim 500-600 \mathrm{~cm}^{-1}\right)$, который связан с взаимодействием „свободных“, но сильно коррелированных, электронов с фононами. При высоких температурах $\sim 67^{\circ} \mathrm{C}$ $(340 \mathrm{~K})$ происходит „перекачка“ интенсивности из узких линий КРС в этот новый разгоревшийся максимум. Данный факт свидетельствует о совершении протяженного по температуре моттовского электронного перехода диэлектрик-металл без изменения моноклинной симметрии решетки.

\section{Заключение}

В настоящей работе продемонстрирована уникальная возможность применения диэлектрической спектроскопии для измерения электрофизических параметров тонких нанокристаллических (около $100 \mathrm{~nm}$ ) пленок сильнокоррелированных материалов и быстрого прецизионного определения их характеристик по температурным и частотным зависимостям $\operatorname{tg} D$. Показано, что фитинг экспериментальных результатов на функцию (1), полученную на основе простой эквивалентной схемы (рис. 3), позволяет определить ключевой параметр наноскристаллических пленок диоксида ванадия: температурную эволюцию ширины запрещенной зоны. Показано также, что с ростом температуры в температурном интервале $0-40^{\circ} \mathrm{C}(273-313 \mathrm{~K})$ происходит „схлопывание“ запрещенной зоны, обусловленное воздействием сильно коррелированных электронов в зоне проводимости на энергетическое положение зон. Появление „свободных“ коррелированных электронов согласуется с данными по КРС, в спектрах которого наблюдается возгорание в этом же температурном интервале максимума, резко отличного по ширине и интенсивности от узких фононных линий спектра КРС изолирующей фазы $\mathrm{VO}_{2}$, который становится доминирующим в металлической фазе при высоких температурах.

Работа выполнена при частичной поддержке Министерства образования и науки РФ (проект № 3.5005.2017/ВУ, РГПУ им. А.И. Герцена).

\section{Список литературы}

[1] Castro R.A., Bordovskĭ V.A., Grabko G.I. // Technic. Phys. Lett. 2010. Vol. 36. P. 783-785.

[2] Nikonorova N.A., Balakina M.Y., Fominykh O.D., Sharipova A.V., Vakhonina T.A., Nazmieva G.N., Castro .A., Yakimansky A.V. // Mater. Chem. Phys. 2016. Vol. 181. P. 217-226.

[3] Castro R.A., Ignatiev A.I., Nikonorov N.V., Sidorov A.I., Stolyarchuk M.V. // J. Non-Crystal. Sol. 2017. Vol. 461. P. 72 79.

[4] Mott N.F. Metal-Insulator Transitions. M.: Nauka, 1979; Londin: Taylor Francis, 1974. 342 c.

[5] Бугаев А.А., Захарченя Б.П., Чудновский Ф.А. Фазовый переход металл - полупроводник и его применение. Л.: Наука, 1979. 183 с.

[6] Bruckner W., Opperman H., Reichelt W., Wolf G., Chudnovsky F.A., Terukov E.I. Vanadium dioxide. Berlin: Academy-Verlag, 1983.

[7] Пат. РФ. № 2575134. Диэлектрический метод диагностики электронных состояний в кристаллах. 2016. А.В. Ильинский, Р.А. Кастро, П.А. Набиуллина, М.Э. Пашкевич, Е.Б. Шадрин.

[8] Ильинский А.В., Шадрин Е.Б. // Письма в ЖТФ. 2001. Т. 27. Вып. 3. С. 54-58.

[9] Ильинский А.В., Квашенкина О.Е., Шадрин Е.Б. // ФТП. 2012. Т. 46. Вып. 9. С. 1194-1208.

[10] Гончарук И.Н., Ильинский А.В., Квашенкина О.Е., Шадрин Е.Б. // ФТТ. 2013. Т. 55. Вып. 1. С. 147-156.

[11] Gatti M., Bruneval F., Olevano V., Reining L. // Phys. Rev. Lett. 2007. Vol. 99. P. 266402-1-4.

[12] Ilinskiy A.V., Kvashenkina O.E., Shadrin E.B.// Smart Nanocomposites. 2013. Vol. 4. P. 109-114.

[13] Мигдал А.Б. // ЖЭТФ. 1957. Т. 32. С. 399.

[14] Шадрин Е.Б., Ильинский А.В. // ФТТ. 2000. Т. 42. Вып. 6. C. 1092. 